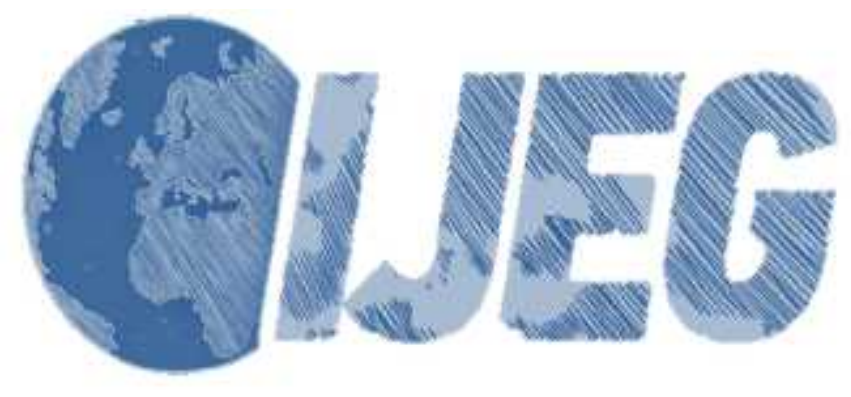

International Journal of Engineering and Geosciences (IJEG), Vol;2, Issue;01, pp. 35-40, February, 2017, ISSN 2548-0960, Turkey, DOI: $\underline{\text { 10.26833/ijeg.287308 }}$

\title{
THE EFFECT OF JPEG COMPRESSION IN CLOSE RANGE PHOTOGRAMMETRY
}

\author{
Akcay, O. ${ }^{1 *}$, Erenoglu, R. C. ${ }^{1}$, Avsar, E. O. ${ }^{1}$ \\ ${ }^{1}$ Canakkale Onsekiz Mart University, Engineering Faculty, \\ Department of Geomatics Engineering, Tel: +90 286 2180018/2201, 17100, Canakkale, Turkey \\ (akcay@comu.edu.tr, ceren@comu.edu.tr, ozguravsar@comu.edu.tr)
}

"Corresponding Author, Received: 13/01/2017, Accepted: 28/01/2017

\begin{abstract}
Digital photogrammetry, using digital camera images, is an important low-cost engineering method to produce precise three-dimensional model of either an object or the part of the earth depending on the image quality. Photogrammetry which is cheaper and more practical than the new technologies such as LIDAR, has increased point cloud generation capacity during the past decade with contributions of computer vision. Images of new camera technologies needs huge storage space due to larger image file sizes. Moreover, this enormousness increases image process time during extraction, orientation and dense matching. The Joint Photographic Experts Group (JPEG) is one of the most commonly used methods as lossy compression standard for the storage purposes of the oversized image file. Particularly, image compression at different rates causes image deteriorations during the processing period. Therefore, the compression rates affect accuracy of photogrammetric measurements. In this study, the close range images compressed at the different levels were investigated to define the compression effect on photogrammetric results, such as orientation parameters and 3D point cloud. The outcomes of this study show that lower compression ratios are acceptable in photogrammetric process when moderate accuracy is sufficient.
\end{abstract}

Keywords: Photogrammetry, JPEG, compression, 3D modeling, image matching, exterior orientation 


\section{INTRODUCTION}

Photogrammetry includes scientific methodologies that calculate the three-dimensional coordinates of an object via measuring corresponding points on the overlapping images. The mathematical relation between an image point and an object point is derived by collinearity equations that based on central projection (Kyle, 2013). The recent integration of computer vision algorithms and photogrammetric methods is leading to interesting procedures which have increasingly automated the entire image-based 3D modelling process (Remondino et al., 2014). In last two decades, Close-Range Photogrammetry (CRP) as a contribution of photogrammetry and computer vision, spread into many fields of engineering applications such as medical modelling applications (Xiao et al., 2014), orthophotos by Unmanned Aerial Systems (UAS) (Akcay, 2015) and documentation of cultural heritages (Y1lmaz et al., 2007). Three dimensional textured models, digital surface models and true orthophotos can be produced using advantages of low-cost CRP software.

CRP software implement automatic point detection and robust matching algorithms for photogrammetric process. The most common algorithm; Scale Invariant Feature Transform (SIFT) is a digital property extraction method that allows automatic identification of characteristic points (Lowe, 2004). Bay et al. (2006), developed Speeded Up Robust Features (SURF), that approximate SIFT algorithm with respect to repeatability, distinctiveness, and robustness, yet can be computed and compared much faster. This is achieved by relying on integral images for image convolutions; by building on the strengths of the leading existing detectors and descriptors by using a Hessian matrixbased measure for the detector, and a distribution-based descriptor. But these algorithms might be inadequate when very high accuracy (sub-pixel) is necessary, such as industrial (Luhmann et al., 2015) and engineering photogrammetric measurements (Avsar et al, 2015). Traditional photogrammetric measurement is to mark corresponding points manually, which requires more time-consuming and labour-intensive process. Furthermore, the accuracy of the manual measurement depends on user experience and therefore required product quality may not be achieved. Third measurement method in CPR is the automatic measurement of specially coded targets within subpixel accuracy. These approximations depend on combined usage of normalised cross correlation and least square image matching. The size and shape of the specially coded targets are determined according to camera-object distance (Y1lmaztürk, 2011).

After image orientation implementation, all three mentioned methods produce three-dimensional model from point cloud by Multi-view stereo (MVS) (Seitz et al., 2006). Multi-view stereo algorithms provide feature detection and are able to construct highly detailed 3D models from multiple images (Furukawa and Ponce, 2007).

Raw data format is the uncompressed or possibly least processed format of the images obtained by digital cameras or scanners. Besides raw formats; camera manufacturers provide lower-file size and high-quality Joint Photographic Experts Group (JPEG) (Hamilton, 1992) and its derivative such as JPEG2000 (Christopouloset al., 2000) images by embedded image processing software in digital camera (Hamilton, 1992). Today, new generation photogrammetric software can process both raw and compressed image files.

Many processing steps applied sequentially while getting JPEG images. First, the colour space conversion is performed. Red, Green, Blue (RGB) image within the visible wavelength color-coded format is converted to $\mathrm{Y}^{\prime} \mathrm{C}_{\mathrm{b}} \mathrm{C}_{\mathrm{r}}\left(\mathrm{Y}^{\prime}\right.$ is luma component and $\mathrm{C}_{\mathrm{b}}, \mathrm{C}_{\mathrm{r}}$ are two chroma components) expressed in the colour space. Colour space conversion, is defined in ITU-R BT.601 standard, previously called CC 601. In second step of the JPEG conversion, especially the colour subsampling process is performed to reduce the data flow rate (Kerr, 2012). In the third step, each channel of the image with the block parsing process is divided into $8 \times 8$ or $16 \times 16$ blocks. The two-dimensional discrete cosine transform (DCT) is applied on each block to determine the energy distribution (Ahmed et al., 1974). Finally, entropy coding with Huffman is calculated for each block (Huffman, 1952) after quantization step is implemented.

Cronk (2001) in his study examined the effect of JPEG compression in CRP. Y1lmaztürk and Akcay (2005) examined the JPEG compression effect on the sub-pixel measurement by using different target sizes. Beside photogrammetric studies, Liang et al. (2006) discussed the effect of compression on remote sensing processes. In the study, the effect of different JPEG image compression ratios on the accuracy of the photogrammetric evaluation and generation of threedimensional point cloud were examined.

\section{METHODOLGY AND APPLICATION}

\subsection{Exterior orientation with different methods}

In order to compare the different photogrammetric measurement methods (manual, SURF and coded targets) explained in the introduction; a statue in the university campus was selected as the study object. The raw images were acquired with a $18 \mathrm{MP}$ Canon EOS 650D without compression. 37 acquired images were evaluated during the study. The images were oriented according to the mentioned methods (Table 1).

Table 1. Comparison of exterior orientation parameters

\begin{tabular}{|c|c|c|c|c|}
\hline \multirow[t]{2}{*}{ Method } & \multicolumn{3}{|c|}{ Mean square error (m) } & \multirow{2}{*}{$\begin{array}{c}\# \\
\text { points } \\
\text { (avg) }\end{array}$} \\
\hline & $X$ & Y & $\mathrm{Z}$ & \\
\hline Manual & 0.00090 & 0.00063 & 0.00084 & 29 \\
\hline SURF & 0.01041 & 0.00654 & 0.00957 & 680 \\
\hline Coded T. & 0.00096 & 0.00066 & 0.00087 & 18 \\
\hline \multirow[t]{2}{*}{ Method } & \multicolumn{3}{|c|}{ Mean square error (degree) } & \\
\hline & Omega & Phi & Карра & \\
\hline Manual & 0.03295 & 0.02586 & 0.03678 & \\
\hline SURF & 0.05423 & 0.04119 & 0.06233 & \\
\hline Coded T. & 0.03409 & 0.02654 & 0.03958 & \\
\hline
\end{tabular}


SURF algorithm is able to define characteristic features which have intensive radiometric and spatial changes (Figure 1). As shown in Table 1; approximately 680 points matched in images with SURF algorithm which is 25-35 times more than two other measurement methods. On the contrary; SURF algorithm delivers mean square error of exterior orientation parameters ten times greater than others.

Similar accuracy results were obtained in orientation when coded targets and manual measurement were compared.

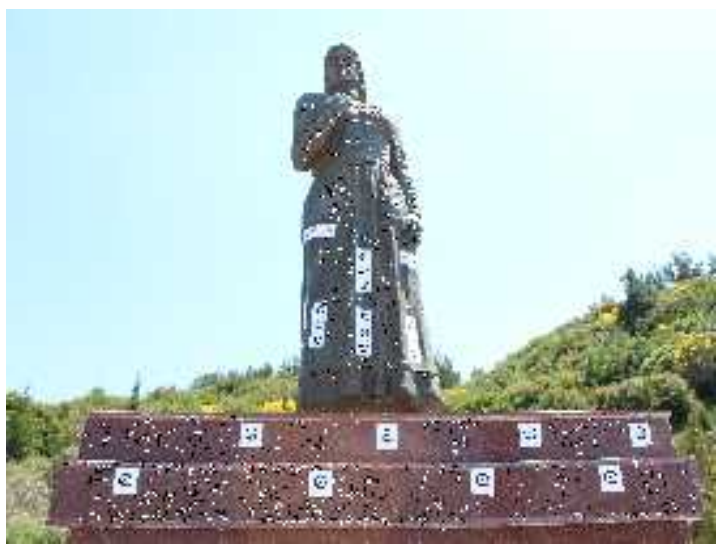

Figure 1. SURF feature extraction results.

\subsection{Application of Image Compression}

The effect of the compression was considered for automatic measurement methods. Therefore, manual measurements were not examined for the compressed images. On the other hand, SURF and coded targets as automatic measurement methods were accounted for different compression levels. Raw data format was converted to JPEG compressed files to obtain ten percentage gradual decreased quality images.

Compression rates especially decreases the amount of the extracted features as well as robust matching number (Chao et al., 2013). However, results showed an unworthy decline in the context of extracted features due to quality loss as shown in Figure 2.

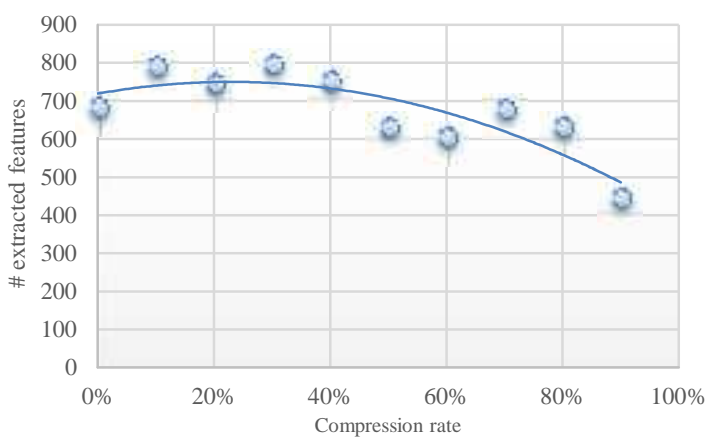

Figure 2. SURF feature extraction results.

Consideration of the extracted features at different compression levels might not be enough in order to infer the influence of the compression. Understanding of the matching performance is also important beside the feature extraction. Low matching performance is clearly seen at more than forty percentage compression rates when Figure 3 is inspected.

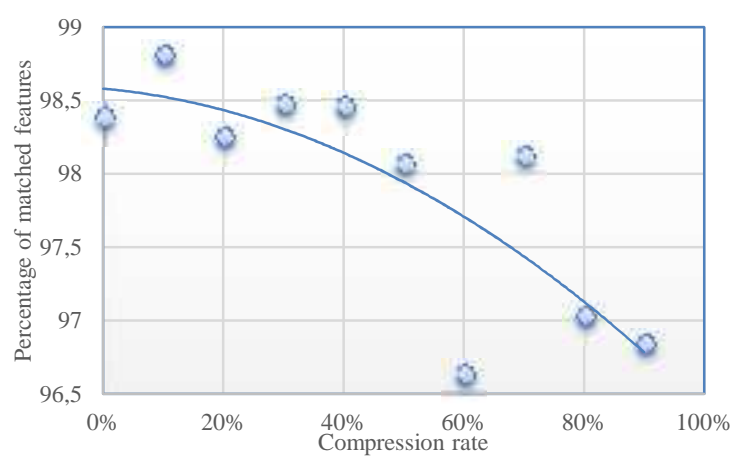

Figure 3. Matched points for SURF.

Coded targets which provide sub-pixel accuracy matching do not give the possibility of an appropriate compression analysis. Automatic photogrammetric measurement with coded target is vulnerable to compression process as the mentioned method is so sensitive to image quality. More than twenty percentage compression makes the photogrammetric calculation failure because of the insufficient matched coded target.

\section{DISCUSSION}

\subsection{Exterior orientation with different methods}

In the study, mean square errors (MSE) of the exterior orientation obtained using automatic measurement methods were also compared at each compression levels. Figure 4 illustrates MSE of the projection centre coordinates $\mathrm{X}_{0}, \mathrm{Y}_{0}, \mathrm{Z}_{0}$ calculated using SURF extraction and matching while Figure 5 indicates MSE of the orientation angles $\varpi, \varphi, \kappa$. As seen in Figure 4, errors of projection centre coordinates sharply increase after forty percent compression level. Figure 5 also indicates that forty percentage compression is determining level during the computation of the rotation angles.



Figure 4. SURF feature extraction results. 


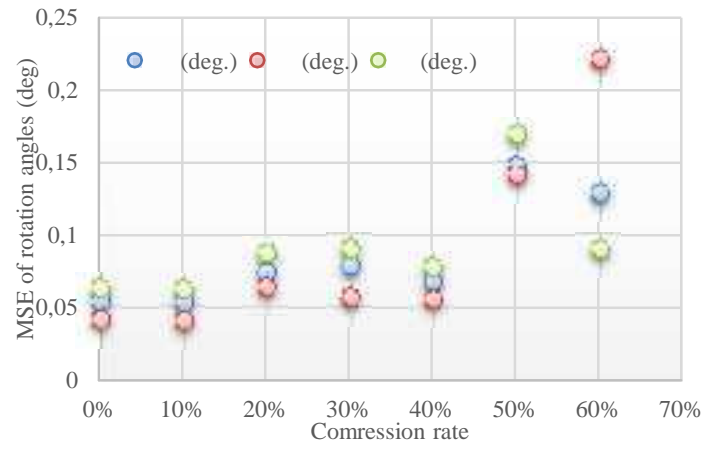

Figure 5. SURF feature extraction results.

An appropriate compression analysis of exterior orientation with coded targets is as not possible as computed with SURF algorithm. Orientations computed with sub-pixel measurement accuracy showed significant deficiencies at low compression rates. Moreover, high compression rates more than twenty percent were not possible to define orientation values. Figures 6 and 7 illustrate that the quality change in images had little effect on orientation accuracy.

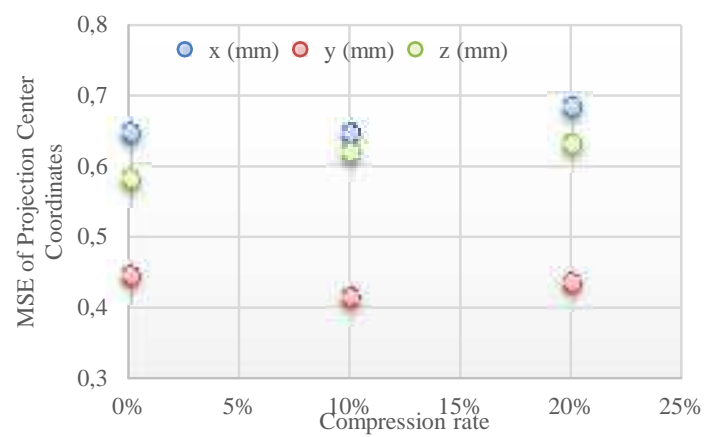

Figure 6. Coded targets feature extraction results.

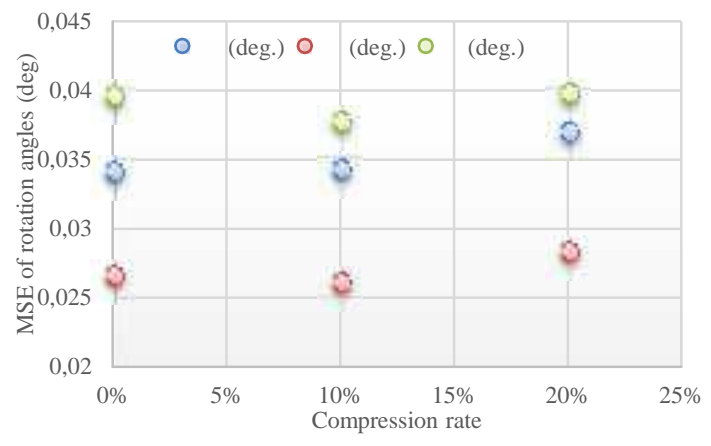

Figure 7. Coded targets feature extraction results.

\subsection{Point cloud generation}

Point cloud generation is implemented with MVS also known as dense matching using multiple images. Accuracy of the obtained point is related with the number and angle of image. Figure 8 shows a part of the point cloud from the statue. As it is seen in the figure, point cloud is so dense. Consequently, the photogrammetric point cloud might be a low-cost alternative to LIDAR point cloud in some cases (Nouwakpo et al., 2015).

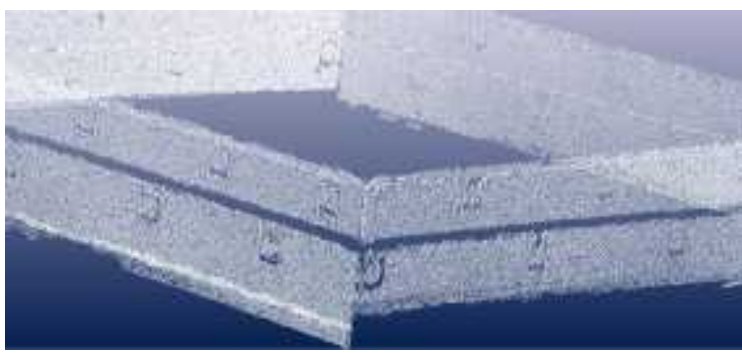

Figure 8. Point Cloud produced by MVS from SURF results.

Applied compression levels also affect results of MVS point cloud beside exterior orientation. This affect emanates from both faulty exterior orientation results and MVS processing with low resolution images. At the high compression levels, gross errors were observed on the point clouds. Figure 9 shows a point cloud which was obtained in fifty percent compression level. The figure explicitly illustrates the failures on the point cloud. However low compression levels less than fifty percent output more stable point clouds as indicated in Figure 10.



Figure 9. Gross errors of point cloud obtained from $\% 50$ compressed images by SURF. 


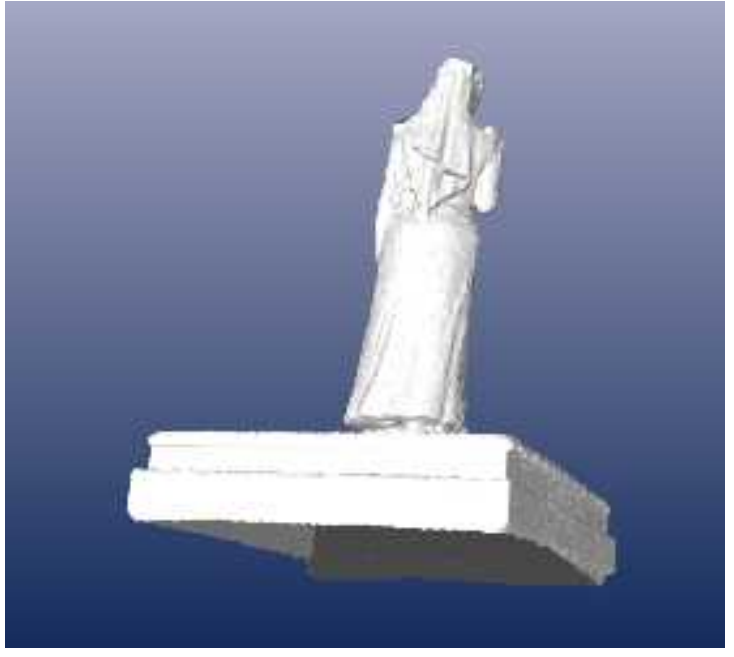

Figure 10. 3D model obtained from $\% 20$ compressed images by SURF.

As MVS results were investigated for each compression level, point numbers generated in the clouds were decreased due to higher compression levels. Particularly, numbers of points diminished at the compression higher than thirty percent. On the contrary, the triangulation and filtered point clouds remained still despite compression (Figure 11). Because triangulation and filtered point cloud were computed independently from images, they were not influenced as much as the original point clouds. Gross errors on the MVS point cloud, also reflect the distortions to the textured models.



Figure 11. Compression-point graph.

\section{RESULTS AND RECOMMENDATIONS}

In the experimental tests, it has been shown that the low compression ratios have a negligible effect on the photogrammetric process. On the other hand, if it is considered that a small amount of compression rates can be seriously reduced in file sizes, up to twenty or thirty percent of compression ratio, the photogrammetric modelling been has proved to be problem-free for the works that do not require high precision. However, sub-pixel measurement method showed bad results with low compressed images as the method needed high resolution.
In the case of high compression ratios, the three orientation methods applied also considerably reduced the precision of the exterior orientation and caused significant rough errors in the $3 \mathrm{D}$ models. It has been observed that the number of point clouds decreases considerably after thirty percent compression.

No colour subsampling is done in JPEG compression performed in this study. By applying the colour subsample in many different combinations, its effect on the photogrammetry can be revealed in future studies. Also in future work, TIFF format and JPEG compression formats, which are obtained without loss from the raw data format, can be compared. Although JPEG is one of the most used standards, the impact on photogrammetric evaluation of other popular image compression algorithms such as JPEG2000 should be discussed.

\section{ACKNOWLEDGMENTS}

This study was supported by Canakkale Onsekiz Mart University, Scientific Research Projects Coordination Unit [grant number FBA-2013-109].

\section{REFERENCES}

Ahmed N., Natarajan T., and Rao K., 1974. Discrete cosine transform, IEEE Trans. Comput., vol. C-23, pp.90 -93 1974.

Akcay, O., 2015. Landslide Fissure Inference Assessment by ANFIS and Logistic Regression Using UAS-Based Photogrammetry. ISPRS International Journal of Geo-Information, 4(4), 2131-2158.

Avsar, E. Ö., Altan, M. O., Doğan, Ü. A., and Akça, D., 2015. Determining Pull-Out Deformations by Means of an Online Photogrammetry Monitoring System. International Journal of Environment and Geoinformatics, 2(1).

Bay, H., Tuytelaars, T., and Van Gool, L., 2006. Surf: Speeded up robust features. In Computer vision-ECCV 2006 pp. 404-417. Springer Berlin Heidelberg.

Chao, J., Chen, H., and Steinbach, E., 2013. On the design of a novel JPEG quantization table for improved feature detection performance. In Image Processing (ICIP), 2013 20th IEEE International Conference on (pp. 1675-1679). IEEE.

Christopoulos, C.A., Skodras, A.N. and Ebrahimi, T., 2000. The JPEG 2000 still image coding system: An overview, IEEE Trans. Consumer Electron, vol. 46, pp.1103 -1127 2000 .

Cronk S., 2001. The Effects of JPEG Image Compression On Digital Close-Range Photogrammetry, Report, Melbourne.

Furukawa Y. and Ponce J., 2007 Accurate, Dense, and Robust Multi-View Stereopsis, CVPR 2007.

Hamilton. E., 1992. JPEG File Interchange Format Version 1.02. C-Cube Microsystems, Sep 1992. 
Huffman, D. A., 1952. A method for the construction of minimum redundancy codes, Proc. IRE, vol. 40, pp.1098 -1101 1952.

Kerr D. A., 2012. Chrominance Subsampling in Digital Images.

http://dougkerr.net/pumpkin/articles/Subsampling.pdf, Jan. 2012.

Kyle, S., 2013. Close-Range Photogrammetry and 3D Imaging; Walter de Gruyter: Berlin, Germany; ISBNISSN: 9783110302783.

Liang Z. , Xinming T. and Lin L., 2006. Effects of JPEG 2000 compression on remote sensing image quality, In Proc. of IEEE International Geoscience and Remote Sensing Symposium, pp. 3297-3300, July 2006.

Luhmann, T., Fraser, C., and Maas, H. G., 2015. Sensor modelling and camera calibration for close-range photogrammetry. ISPRS Journal of Photogrammetry and Remote Sensing.

Lowe, D. G., 2004. Distinctive image features from scale-invariant keypoints., International Journal of Computer Vision, 60, 2, pp. 91-110.

Nouwakpo, S. K., Weltz, M. A., and McGwire, K., 2015. Assessing the performance of structure from motion photogrammetry and terrestrial LiDAR for reconstructing soil surface microtopography of naturally vegetated plots. Earth Surface Processes and Landforms. 41(3) 308-322.

Remondino, F., Spera, M.G., Nocerino, E., Menna, F. and Nex, F., 2014. State of the art in high density image matching. Photogramm. Rec., 29, 144-166.

Seitz, S. M., Curless, B., Diebel, J., Scharstein, D., and Szeliski, R., 2006. A comparison and evaluation of multi-view stereo reconstruction algorithms. In Computer vision and pattern recognition, 2006 IEEE Computer Society Conference on, 1, 519-528. IEEE.

Xiao, K., Zardawi, F., van Noort, R., and Yates, J. M., 2014. Developing a 3D colour image reproduction system for additive manufacturing of facial prostheses. The International Journal of Advanced Manufacturing Technology, 70(9-12), 2043-2049.

Yilmaz, H. M., Yakar, M., Gulec, S. A., and Dulgerler, O. N., 2007. Importance of digital close-range photogrammetry in documentation of cultural heritage. Journal of Cultural Heritage, 8(4), 428-433.

Y1lmaztürk, F., 2011. Full-automatic self-calibration of color digital cameras using color targets. Optics express, 19(19), 18164-18174.

Y1lmaztürk F., Akçay Ö., 2005. Jpeg Görüntü Sıkıştırmanın Yakın Mesafe Dijital Fotogrametri Üzerindeki Etkileri, s. 918-925, 10. Türkiye Harita Bilimsel ve Teknik Kurultayı, Ankara, Türkiye, 28.03.2005 - 01.04.2005.
Copyright (C) International Journal of Engineering and Geosciences (IJEG). All rights reserved, including the making of copies unless permission is obtained from the copyright proprietors. 